\title{
The Energy Dependence of the Underlying Event in Hadronic Collisions
}

\author{
Rick Field \\ (For the CDF Collaboration) \\ Department of Physics, University of Florida \\ Gainesville, Florida, 32611, USA \\ E-mail: rfield@phys.ufl.edu
}

At CDF we study charged particle production ( $\mathrm{p}_{\mathrm{T}}>0.5 \mathrm{GeV} / \mathrm{c},|\eta|<0.8$ ) in proton-antiproton collisions at $300 \mathrm{GeV}, 900 \mathrm{GeV}$, and $1.96 \mathrm{TeV}$. The $300 \mathrm{GeV}$ and $900 \mathrm{GeV}$ data are a result of the "Tevatron Energy Scan” which was performed just before the Tevatron was shut down. We use the direction of the leading charged particle in each event, PTmax, to define three regions of $\eta-\phi$ space; “toward”, "away”, and "transverse”. The "transverse” region is further divided into the "transMAX" and "transMIN" contributions. The "transMIN" region is very sensitive to the multiple parton interaction component (MPI) of the "underlying event", while the "transDIF" ("transMAX” minus "transMIN") is very sensitive to the initial and final-state radiation. This CDF analysis together with LHC data provides a detailed study the energy dependence of the various components of the "underlying event" in hadronic collisions.

The European Physical Society Conference on High Energy Physics

18-24 July, 2013

Stockholm, Sweden 
Min-bias (MB) is a generic term which refers to events that are selected with a "loose" trigger that accepts a large fraction of the overall inelastic cross section. The CDF MB trigger requires at least one charged particle in the forward region $3.2<\eta<5.9$ and simultaneously at least one charged particle in the backward region $-5.9<\eta<-3.2$, where the pseudo-rapidity $\eta=$ $-\log \left(\tan \left(\theta_{\mathrm{cm}} / 2\right)\right)$. The underlying event (UE) consists of the beam-beam remnants (BBR) and the multiple parton interactions (MPI) that accompany a hard scattering. The UE is an unavoidable background to hard-scattering collider events. To study the UE we use MB data, however, MB and UE are not the same object. The majority of MB collisions are "soft", while the UE is studied in events in which a hard-scattering has occurred. One uses the structure of the hard hadron-hadron collision to experimentally study the UE. As illustrated in Figure 1, on an eventby-event bases, a "leading object" is used to define regions of $\eta-\phi$ space, where $\eta$ is the pseudorapidity and $\theta_{\mathrm{cm}}$ is the center-of-mass polar scattering angle [1,2]. Here we use the highest transverse momentum charged particle in the event, PTmax, as the leading object. On an event by event basis, we define "transMAX" ("transMIN") to be the maximum (minimum) number of charged particles or the scalar $\mathrm{p}_{\mathrm{T}}$ sum of charged particles in the two "transverse" regions, $60^{\circ}<$ $\Delta \phi<120^{\circ},|\eta|<\eta_{\text {cut }}$ and $60^{\circ}<-\Delta \phi<120^{\circ},|\eta|<\eta_{\text {cut. }}$ Densities are then formed by dividing by the area in $\eta-\phi$ space. "TransMAX" and "transMIN" each have an area of $\Delta \eta \Delta \phi=2 \eta_{\text {cut }} \times 2 \pi / 6$, where we take $\eta_{\text {cut }}=0.8$. The overall "transverse" region (i.e. "transAVE") is the average of the "transMAX” and the "transMIN” regions.

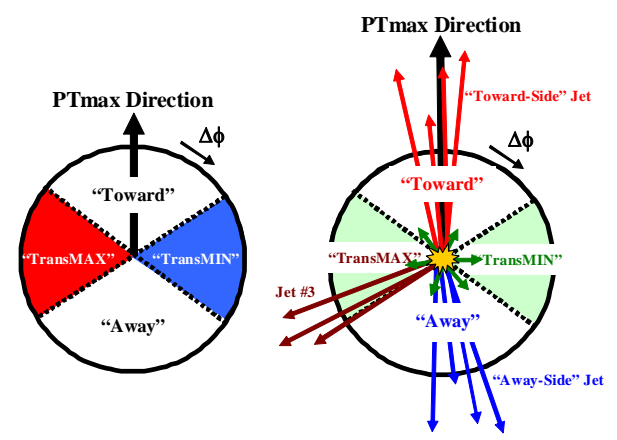

Figure 1. (left) Illustration of correlations in azimuthal angle $\Delta \phi$ relative to the direction of the leading charged particle in the event, PTmax. The relative angle $\Delta \phi=\phi-\phi_{\text {MAX }}$, where $\phi_{\text {MAX }}$ is the azimuthal angle of PTmax and $\phi$ is the azimuthal angle of a charged particle. On an event by event basis, we define "transMAX" ("transMIN") to be the maximum (minimum) of the two "transverse" regions, $60^{\circ}<\Delta \phi<120^{\circ},|\eta|<\eta_{\text {cut }}$ and $60^{\circ}<-\Delta \phi<120^{\circ}$, $|\eta|<\eta_{\text {cut }}$, where we take $\eta_{\text {cut }}=0.8$. The overall "transverse" region (i.e. "transAVE") is the average of the "transMAX" and the "transMIN" regions. (right) llustration of the topology of a hadron-hadron collision in which a "hard" parton-parton collision has occurred. The "toward" region contains the leading "jet", while the "away" region, on the average, contains the "away-side" "jet". For events with large initial or final-state radiation the "transMAX" region contains the third jet, while both the "transMAX" and "transMIN" regions receive contributions from the MPI and beam-beam remnants. Thus, the "transMIN" region is very sensitive to the MPI and beam-beam remnants, while the "transMAX" minus the "transMIN" (i.e. "transDIF") is very sensitive to initial and final-state radiation.

Figure 1 illustrates the topology of a hadron-hadron collision in which a "hard" partonparton collision has occurred. The "toward" region contains the leading "jet", while the "away" region, on the average, contains the "away-side" "jet". For events with large initial or final-state radiation the "transMAX" region contains the third jet, while both the "transMAX" and "transMIN" regions receive contributions from the MPI and beam-beam remnants. Thus, the "transMIN" region is very sensitive to the MPI and beam-beam remnants, while "transDIF" ("transMAX" minus the "transMIN") is very sensitive to initial and final-state radiation [3]. 


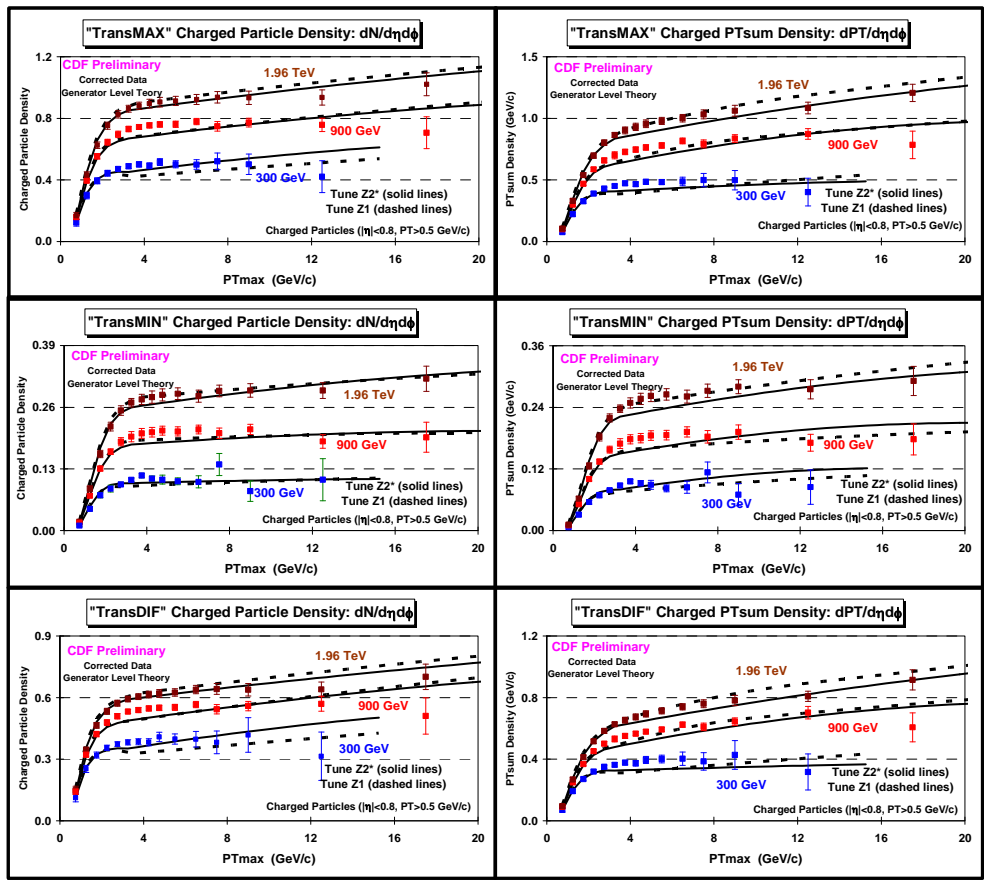

Figure 2. Preliminary CDF data at $1.96 \mathrm{TeV}, 900 \mathrm{GeV}$, and $300 \mathrm{GeV}$ on the "transMAX" charged particle density (top left), the "transMAX" charged PTsum density (top right), the "transMIN" charged particle density (middle left), the "transMIN" charged PTsum density (middle right), the "transDIF" charged particle density (bottom left), and the "transDIF" charged PTsum density (bottom right) as defined by the leading charged particle, PTmax, as a function of PTmax. The "transDIF" density is equal to the "transMAX" density minus the "transMIN" density. The charged particles have $\mathrm{p}_{\mathrm{T}}>0.5 \mathrm{GeV}$ and $|\eta|<0.8$. The data are corrected to the particle level with errors that include both the statistical error and the systematic uncertainty and are compared with PYTHIA 6.4 Tune Z1 and Tune Z2* at the generator level.

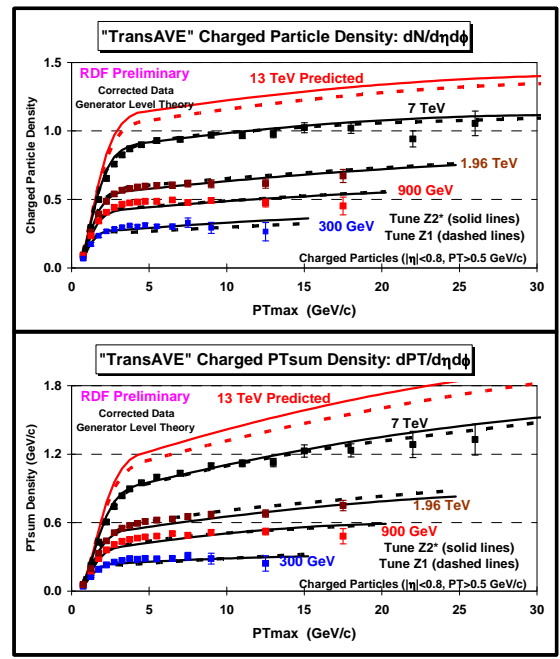

Figure 3. Preliminary CMS data [9] at $7 \mathrm{TeV}$ and preliminary CDF data at $1.96 \mathrm{TeV}, 900 \mathrm{GeV}$, and $300 \mathrm{GeV}$ on the "transAVE" charged particle density (top) and the "transAVE" charged PTsum density (bottom) as defined by the leading charged particle, PTmax, as a function of PTmax. The "transAVE" density is equal to the average of the "transMAX" density and the "transMIN" density (i.e. overall "transverse" density). The charged particles have $\mathrm{p}_{\mathrm{T}}>$ $0.5 \mathrm{GeV}$ and $|\eta|<0.8$. The data are corrected to the particle level with errors that include both the statistical error and the systematic uncertainty and are compared with PYTHIA 6.4 Tune Z1 and Tune Z2* at the generator level. The predictions at $13 \mathrm{TeV}$ are also shown.

Figure 2 shows the preliminary $\mathrm{CDF}$ data at $1.96 \mathrm{TeV}, 900 \mathrm{GeV}$, and $300 \mathrm{GeV}$ on the "transMAX”, "transMIN", and "transDIF" charged particle and PTsum densities as defined by the leading charged particle, PTmax, as a function of PTmax. The charged particles have $\mathrm{p}_{\mathrm{T}}>$ 
$0.5 \mathrm{GeV}$ and $|\eta|<0.8$. The data are corrected to the particle level with errors that include both the statistical error and the systematic uncertainty and are compared with PYTHIA 6.4 Tune Z1 and Tune Z2* at the generator level. QCD Monte-Carlo generators such as PYTHIA [4] have parameters which may be adjusted to control the behavior of their event modeling. A specified set of these parameters that has been adjusted to better fit some aspects of the data is referred to as a "tune" [5,6]. Tune Z1 (CTEQ5L) and Tune Z2* (CTEQ6L) are PYTHIA 6.4 tunes that were constructed by fitting CMS UE data at $900 \mathrm{GeV}$ and $7 \mathrm{TeV}[7,8]$. Both tunes do a fairly good (although not perfect) job in describing the CDF UE data at $1.96 \mathrm{TeV}, 900 \mathrm{GeV}$, and 300 $\mathrm{GeV}$.

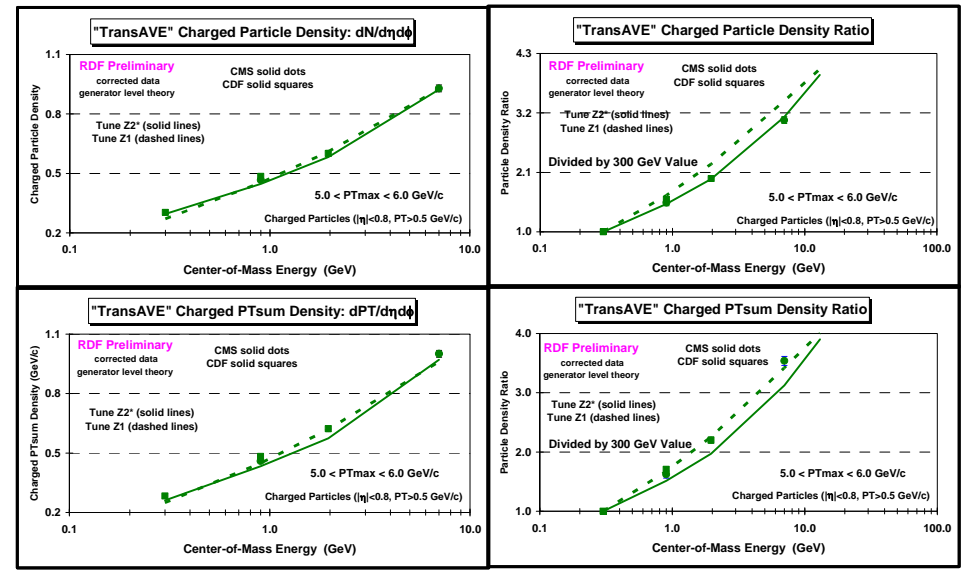

Figure 4. (left column) Preliminary CMS data [9] at $7 \mathrm{TeV}$ and $900 \mathrm{GeV}$ (solid squares) and preliminary CDF data at $1.96 \mathrm{TeV}, 900 \mathrm{GeV}$, and $300 \mathrm{GeV}$ (solid dots) on the "transAVE" charged particle density (top) and the "transAVE" charged PTsum density (bottom) as defined by the leading charged particle, PTmax, for $5.0<$ PTmax < $6.0 \mathrm{GeV} / \mathrm{c}$ versus the center-of-mass energy (log scale). The "transAVE" density is equal to the average of the "transMAX" density and the "transMIN" density (i.e. overall "transverse" density). (right column) Ratio of the data to the corresponding value at $300 \mathrm{GeV}$ for the "transAVE" charged particle density (top) and the "transAVE" charged PTsum density (bottom) as defined by the leading charged particle, PTmax, for $5.0<$ PTmax $<6.0 \mathrm{GeV} / \mathrm{c}$ versus the center-of-mass energy $\left(\log\right.$ scale). The charged particles have $\mathrm{p}_{\mathrm{T}}>0.5 \mathrm{GeV}$ and $|\eta|<0.8$. The data are corrected to the particle level with errors that include both the statistical error and the systematic uncertainty and are compared with PYTHIA 6.4 Tune Z1 and Tune Z2* at the generator level extrapolated to $13 \mathrm{TeV}$ (right column).

Figure 3 shows preliminary CMS data [9] at $7 \mathrm{TeV}$ together with preliminary CDF data at $1.96 \mathrm{TeV}, 900 \mathrm{GeV}$, and $300 \mathrm{GeV}$ for the "transAVE" charged particle and PTsum densities as defined by the leading charged particle, PTmax, as a function of PTmax. The "transAVE" density is equal to the average of the "transMAX" density and the "transMIN" density (i.e. overall "transverse" density). Figure 4 shows preliminary CMS data [9] at $7 \mathrm{TeV}$ and $900 \mathrm{GeV}$ together with $\mathrm{CDF}$ data at $1.96 \mathrm{TeV}, 900 \mathrm{GeV}$, and $300 \mathrm{GeV}$ for the "transAVE" charged particle and PTsum densities as defined by the leading charged particle, PTmax, for $5.0<$ PTmax $<6.0 \mathrm{GeV} / \mathrm{c}$ versus the center-of-mass energy. Figure 4 also shows the ratio of the data to the corresponding value at $300 \mathrm{GeV}$ for the "transAVE" charged particle and PTsum densities. The "transAVE" charge particle density increases by a factor of about 3.0 in going from $300 \mathrm{GeV}$ to $7 \mathrm{TeV}$, while the PTsum density increase by a factor of about 3.5. This is a reflection of the fact that the "transverse" average $\mathrm{p}_{\mathrm{T}}$ of the charged particles is increasing. Both Tune Z1 and Tune Z2* do a fairly good (although not perfect) job in describing the energy dependence of "transAVE”. 


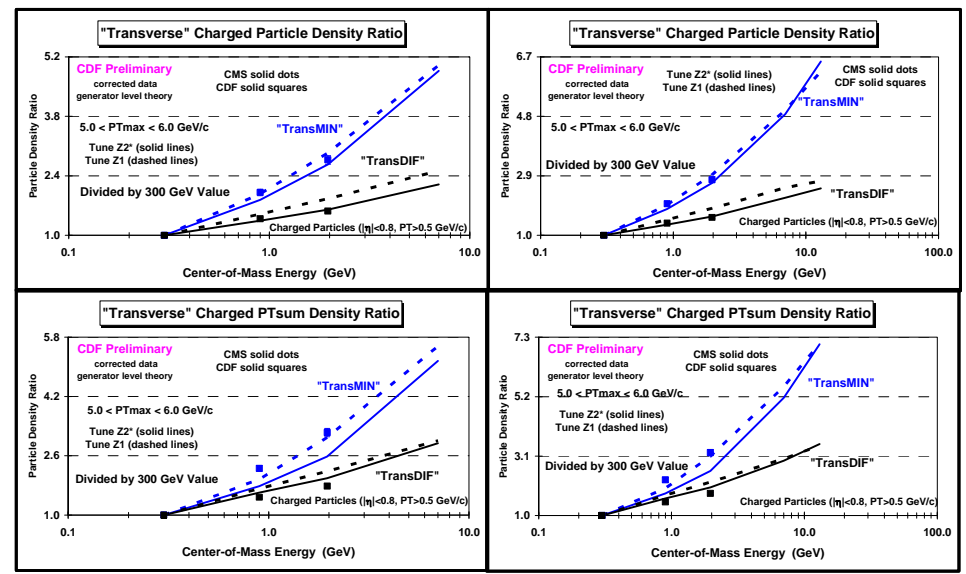

Figure 5. Preliminary CDF data at $1.96 \mathrm{TeV}, 900 \mathrm{GeV}$, and $300 \mathrm{GeV}$ on the "transMIN" and "transDIF" charged particle density (top row) and the "transMIN" and "transDIF" charged PTsum density (bottom row) as defined by the leading charged particle, PTmax, for $5.0<$ PTmax $<6.0 \mathrm{GeV} / \mathrm{c}$ versus the center-of-mass energy $(\log$ scale). The plots show the ratio of the data to the corresponding value at $300 \mathrm{GeV}$. The charged particles have $\mathrm{p}_{\mathrm{T}}>0.5 \mathrm{GeV}$ and $|\eta|<0.8$. The data are corrected to the particle level with errors that include both the statistical error and the systematic uncertainty and are compared with PYTHIA 6.4 Tune Z1 and Tune Z2* at the generator level extrapolated to $7 \mathrm{TeV}$ (left column) and $13 \mathrm{TeV}$ (right column).

Figure 5 shows the energy dependence of the "transMIN" and "transDIF" components. The "transMIN" density (more sensitive to MPI \& BBR) increases much faster with center-ofmass energy than does the "transDIF" density (more sensitive to ISR \& FSR). The MPI increases like a power of the center-of-mass energy, while the ISR \& FSR increase logarithmically. Tune Z2* predicts that the "transMIN" charged particle density increases by factor of around 6.6 in going from $300 \mathrm{GeV}$ to $13 \mathrm{TeV}$, while the "transDIF" charged particle density is predicted to increase by only a factor of around 2.5. This is the first time we have seen the different energy dependences of these two components. Previously we only had information on the energy dependence of the "transAVE" charge particle density. Both Tune $\mathrm{Z} 1$ and Tune Z2* do a fairly good (although not perfect) job in describing the energy dependence of "transMIN" and "transDIF". What we are learning will allow for a deeper understanding of the BBR and MPI which will result in more precise predictions at the future LHC energies of 13 and $14 \mathrm{TeV}$.

\section{References}

[1] The CDF Collaboration, Charged Jet Evolution and the Underlying Event in Proton-Antiproton Collisions at 1.8 TeV, Phys. Rev. D65, 092002 (2002).

[2] R. Field, The Underlying Event in Hadronic Collision, Annual Review of Nuclear and Particle Science, 62, 427-457 (2012).

[3] Using "transMAX" and "transMIN" was first suggested by Bryan Webber and implemented in a paper by Jon Pumplin, Hard Underlying Event Corrections to Inclusive Jet Cross-Sections, Phys. Rev. D57, 5787 (1998).

[4] T. Sjöstrand, Phys. Lett. 157B, 321 (1985); M. Bengtsson, T. Sjöstrand, and M. van Zijl, Z. Phys. C32, 67 (1986); T. Sjöstrand and M. van Zijl, Phys. Rev. D36, 2019 (1987). T. Sjöstrand, P. Eden, C. Friberg, L. Lonnblad, G. Miu, S. Mrenna and E. Norrbin, Computer Physics Commun. 135, 238 (2001). 
[5] R. Field, CDF Run 2 Monte-Carlo Tunes, Tevatron-for-LHC: Report of the QCD Working Group, arXiv:hep-ph/0610012, FERMILAB-Conf-06-359, October 1, 2006.

[6] P. Skands, The Perugia Tunes, 2009. arXiv:0905.3418.

[7] The CMS Collaboration, Measurement of the Underlying Event Activity at the LHC at 7 TeV and Comparison with 0.9 TeV, J. High Energy Phys. 09 (2011) 109, arXiv:1107.0330.

[8] R. Field, Min-Bias and the Underlying Event at the LHC, arXiv:1110.5530, proceedings of the $51^{\text {st }}$ Cracow School of Theoretical Physics: The Soft Side of the LHC, Zakopane, June 11 - 19, 2011, Acta Physica Polonica B42, 2631 (2011).

[9] The CMS Collaboration, Measurement of the Underlying Event Activity in Proton-Proton Collisions at the LHC using Leading Tracks at $7 \mathrm{TeV}$ and Comparison with $0.9 \mathrm{TeV}$, CMS PAS FSQ-12-020 (2012). 